\title{
Measuring the Atmospheric Influence on Differential Astrometry: a Simple Method Applied to Wide Field CCD Frames
}

\author{
N. Zacharias ${ }^{1}$ \\ U.S. Naval Observatory, 3450 Mass. Ave. N.W., Washington D.C. 20392, \\ Electronic mail: nz@pyxis.usno.navy.mil
}

Received —

PASP revised manuscript, 12 Aug 96, proofs to NZ

\footnotetext{
${ }^{1}$ with Universities Space Research Association (USRA), Division of Astronomy and Space Physics, Washington D.C., based on observations made at KPNO and CTIO
} 


\begin{abstract}
Sets of short exposure, guided CCD frames are used to measure the noise added by the atmosphere to differential astrometric observations. Large nightly variations that are correlated with the seeing have been found in the data obtained over 2 years at the KPNO and CTIO 0.9-meter telescopes. The rms noise added by the atmosphere, after a linear transformation of the raw $x, y$ data, is found to be 3 to 7 mas, normalized to 100 seconds exposure time and a field of view of 20 arcminutes near the zenith. An additional nearly constant noise (base-level) of 8.5 mas $=0.012$ pixel is found for the KPNO and 6.0 mas $=0.015$ pixel for the CTIO telescope. This implies that a ground-based, all sky, astrometric survey from guided CCD frames is more likely limited by the base-level noise than by the atmosphere and could reach an accuracy better than 10 mas under good seeing conditions.
\end{abstract}

Subject headings: astrometry: limits by the atmosphere, guided CCD frames 


\section{INTRODUCTION}

Turbulence in the Earth's atmosphere adds noise to ground-based astrometric observations. Semi-empirical and empirical results have been published previously, e.g. (Lindegren 1980; Kleine 1983; Han 1989; Monet and Monet 1992, Han and Gatewood 1995). This effect ultimately limits the accuracy of ground-based astrometric observations, and it is important to find these limits. The effect is largest, about 100 mas, for absolute astrometry. For differential astrometry, previous investigations have dealt with angular separation measures. The effect is found to be at the 1-2 mas level for arcminute separations and several minutes integration time (Han and Gatewood 1995), e.g. applicable to double star and parallax observations.

Here we will go one step further and define $\sigma_{a t m}$ as the added noise introduced by the atmosphere to astrometric observations, after an orthogonal or linear mapping model has been applied to the $x, y$ data of guided exposures. This is more appropriate for astrometric imaging observations, because such a mapping model is used for the calibration of the $x, y$ data to the reference star positions anyway, thus absorbing terms like scale factor and field rotation. The proposed technique in principle can be used with photographic plates as well as with CCD imaging, although the use of CCDs is more likely to show any atmospheric effect due to usually shorter exposure times and higher internal precision.

The dependence of $\sigma_{a t m}$ on integration time is well known to be $\sigma_{a t m} \sim t^{-1 / 2}$ and we assume this relationship here in our definition of $\sigma_{a t m}$. The dependence of $\sigma_{a t m}$ on the field of view (FOV) is approximately known to be $\sigma_{a t m} \sim(F O V)^{-1 / 3}$ (e.g. Han 1989), at least for fields smaller than about half a degree, and will not be investigated here. Our goal is to determine the range of $\sigma_{a t m}$ for different nights and atmospheric conditions and look for a dependence on seeing, as determined from the full width at half maximum (FWHM) of the image profiles. 


\section{METHOD}

A simple method is introduced here to measure $\sigma_{a t m}$ based on direct CCD imaging without the need for further instrumentation. CCD frames have been taken of fields with a high star density and reduced by standard procedures including bias removal and flat-fielding. Circular symmetric 2-dimensional Gaussian image profiles have been fitted by least-squares methods to the flat-fielded CCD pixel data. Fig. 1 gives an example for the standard error in position plotted vs. instrumental magnitude for individual images. Stars within a dynamic range from the saturation limit (here set to 10th magnitude) to about 5 magnitudes fainter, display an almost constant level of precision for the $x, y$ position as obtained by the image profile fit. The positional error increases for fainter stars because of the smaller $\mathrm{S} / \mathrm{N}$ ratio and for brighter stars because of the model insufficiencies (saturated pixels, diffraction spikes). Images well above the average position error for their magnitudes are either from double stars or galaxies and have been excluded from this investigation. This diagram does not change with exposure time, except for a shift along the magnitude scale and the number of images available in a given range of instrumental magnitudes.

Assume 2 CCD frames of equal exposure times have been taken within a short period of time under the same conditions (atmosphere and telescope). The field center of the second exposure has been shifted by a few pixels with respect to the first one. Thus, independent observations have been obtained with images of the same star located on different pixels of the CCD for both frames. Only the repeatability of the observations is investigated here, so no attempt has been made to convert the $x, y$ measures into right ascensions and declinations. All error contributions related to field distortions are avoided because the same approximate field center has been used for both exposures.

The $x, y$ coordinates of the first frame are transformed into the system of the $x, y$ coordinates of the second frame with a least-squares fit using either a linear or orthogonal 
model. Only those stars within the magnitude range of almost constant fit precision as described above, have been used for this transformation. The variance of the transformation between the 2 exposures, $\sigma_{\text {trans }}^{2}$, is

$$
\sigma_{\text {trans }}^{2}=2\left(\sigma_{\text {atm }}^{2}+\sigma_{b}^{2}\right)
$$

with $\sigma_{a t m}$ being the contribution from the atmosphere and $\sigma_{b}$ the remaining error contribution - the base-level - as inherent in our procedure and instrument (model insufficiencies, digitization errors, etc.), for each individual CCD frame. Defining $\sigma_{a}$ from $\sigma_{a t m}=\sigma_{a} t^{-1 / 2}$ with exposure time $t$ in seconds we arrive at

$$
\sigma_{\text {trans }}^{2} / 2=\sigma_{a}^{2} t^{-1}+\sigma_{b}^{2}=\sigma^{2}
$$

which is a linear relationship between the observable quantity $\sigma^{2}$ and the nearly error free parameter $t^{-1}$. Assuming constant observational conditions for the time to take more sets of CCD frame pairs for other exposure times, we can solve for $\sigma_{a}$ and $\sigma_{b}$.

\section{OBSERVATIONS}

Observing runs for the Radio-Optical Reference Frame (RORF) project (Johnston et al. 1991) have been conducted from 1994 to 1996 at the 0.9-meter telescopes on Kitt Peak and Cerro Tololo (Zacharias et al. 1995). The KPNO 0.9-meter telescope has a scale of 0.68 " / pixel and a FOV of 23.2', while those numbers are $0.40 "$ / pixel and 13.6 ' for the CTIO telescope.

A few CCD frames were specifically taken to investigate the limits set by the atmosphere on astrometric accuracy. Fields with a high star density (close to the Milky Way) and close to the zenith, if possible, were observed close to the meridian. For most of 
the selected fields, 2 frames of 40, 20 and 10 seconds exposure time each were taken with the same Gunn $\mathrm{r}$ filter in addition to the long exposures for the RORF project. An off-axis autoguider was used with guide stars selected close to the edge of the main FOV. The integration and correction cycle time was set to about 1 second and the system was guiding for at least 10 seconds before the start of a new exposure.

Figure 2 shows an example of $\sigma^{2}$ plotted vs. $t^{-1}$ for the 4 exposure times. A linear model has been used for the $x, y$ transformations. The filled circles and open squares are the results for the $x$ and $y$ coordinates respectively (along $\delta, \alpha$ for the KPNO telescope).

Because there are more faint than bright stars, the longer exposure frames show more stars near the saturation limit than the short exposure frames. Also, for a given constant number of stars used for different transformations, the value of $\sigma_{\text {trans }}$ is better determined for longer exposure times because of the smaller scatter in the $x, y$ transformation data due to better image definition from the longer integration time. Thus, weights have been assigned to each measured $\sigma_{\text {trans }}^{2}$ value. Let $E(y)$ be an estimate of the standard error of the quantity $y$ and $y=x^{2}$ with $x=\sigma_{\text {trans }}$, then we have from the error propagation law

$$
E(y)=E\left(x^{2}\right)=2 x E(x)
$$

$E(x)$ is here the standard error of the mean, using all $n_{\text {stars }}$ star pairs for the transformation, thus

$$
E(x)=\sigma_{\text {trans }} / \sqrt{n_{\text {stars }}}
$$

Putting everything together we arrive at the adopted formula

$$
\text { error estimate on } \sigma_{\text {trans }}^{2}=2 \sigma_{\text {trans }} \frac{\sigma_{\text {trans }}}{\sqrt{n_{\text {stars }}}}
$$


The weighting is not critical for the determination of the slope itself, i.e. for the atmospheric contribution. The determination of the base-level and the error estimates on the results depend more strongly on the choice of the weighting algorithm. This conclusion was obtained from tests made with different weighting algorithms, including unweighted reductions.

A weighted least-squares fit was performed with the $\sigma^{2}$ vs. $t^{-1}$ data points, in order to obtain the slope and constant of the straight line predicted by the theory. Fit results for each axis separately (dotted, full line) as well as for the combined data (dashed line), are shown in Fig. 2.

\section{RESULTS}

From the straight line fit of the $\sigma^{2}$ vs. $t^{-1}$ plots for the combined data of both axes, $\sigma_{a}$ and $\sigma_{b}$, and their errors were calculated. Table 1 lists all observations and results. The first line for each observation shows the result from the linear transformation model, while the second line shows the result as obtained with the orthogonal model. The last

column displays $\sigma_{a n}$, which is $\sigma_{a}$ normalized to 100 seconds exposure time and a FOV of 20 arcminutes for the zenith, obtained from

$$
\sigma_{a n}=\sigma_{a}\left(\frac{1^{s}}{100^{s}}\right)^{1 / 2}\left(\frac{20^{\prime}}{f o v}\right)^{1 / 3} \cos z
$$

with fov being the field of view in arcminutes as used for the $x, y$ transformations and $z$ being the mean zenith distance while observing the field. Fig. 3 shows results for $\sigma_{a n}$ obtained with the linear $x, y$ transformation model plotted vs. FWHM, scaled to the zenith with a $\cos z$ factor, adopted from (Lindegren 1980). 


\section{DISCUSSION}

There is a large nightly variation in the atmospheric influence on the observed star positions, which is correlated with seeing (FWHM), but "the seeing value" alone does not determine $\sigma_{a n}$. On the average we obtain $\sigma_{a n} \approx 3$ mas and 6 mas for 1 and 2 arcsecond seeing respectively.

The results obtained with the orthogonal $x, y$ transformation model give on the average larger numbers for $\sigma_{a n}$ by about $10 \%$. This is expected, because fewer parameters will model less of the real atmospheric effects.

All our results hold only for this type of guided imaging observing procedure. For differential transit circle or scanning mode observations, the modelling of the atmospheric effects is different, as will be the residual effects caused by the atmosphere on the astrometric results (Stone et al. 1996).

Lindegren (1980) obtained standard errors for observing the separation of stars, i.e. a different kind of differential astrometry from that investigated here. His result, scaled to 100 seconds exposure time and a mean separation of 10' (comparable to our 20' FOV), is about 19 mas. Results by Han (1989) would lead to 14 mas for this case. Both Lindegren's and Han's results are obtained in medium seeing conditions $(\approx 2$ "), thus they have to be compared to our 6 mas, which is a factor of 2 to 3 smaller. Scaled to a 100 second exposure time and a star separation of 10', Han and Gatewood (1995) found $\sigma_{a n}=5.4$ mas from star trail observations obtained from Mauna Kea. Our result is 3 mas for good seeing, which is smaller by almost a factor of 2 .

Separation measurements made at the 61 -inch Flagstaff telescope result in an

atmospheric contribution of 9 to 28 mas for this case, depending on the night (Monet and Monet 1992, Monet 1996). Again our result is a factor of 2 to 3 smaller. Similar 
to our results, Monet and Monet find a lose correlation with seeing, which ranged from FWHM=1.2" to 2.3". According to a hypothesis (Monet 1996), local effects near the dome cause some of the "astrometric seeing", not correlated with the general FWHM.

This difference between our results and those obtained by other investigations can be explained by the different types of observations. The simultaneous observation of all stars in the FOV seems to be important. Moreover, our guided exposures follow correlated image motions of a star field, caused by the atmosphere, and thus reduce the noise contribution compared to other differential observing procedures. Also, a linear reduction model will give smaller residuals as compared to angular separation measurements with fewer free parameters.

As a by-product of this method, the base-level of accuracy has been determined as well. The mean of all $\sigma_{b}$ with a standard error smaller than 1.0 mas is found to be 8.5 mas $=$ 0.012 pixel for all KPNO observations. The corresponding result for the CTIO instrument is 6.0 mas $=0.015$ pixel. These numbers are for a single observation per coordinate. The slightly smaller value for $\sigma_{b}$ (in pixel units) for the KPNO instrument can be explained by the better optical quality of that telescope, which includes a field flattener corrector lens. Imperfections in the CCD, the optics of the telescope and model deficiencies contribute to this base-level of astrometric accuracy, which is under further investigation (Zacharias and Rafferty 1995; Winter 1996; Zacharias 1997).

\section{CONCLUSIONS}

A large nightly variation (factor of 2) of the noise added by the atmosphere to differential astrometry has been found. This added noise is correlated with the seeing. In good seeing conditions $(\approx 1 ")$ the contribution of the atmosphere to differential astrometry 
can be as small as 3 mas for guided 100-second exposures and a FOV of 20 arcminutes for 0.9-meter aperture telescopes.

Guided exposures with simultaneous observation of all stars in the FOV give a considerable (about a factor of 2) advantage over angular separation measurements made with other differential astrometric observing techniques.

For a 1 degree FOV astrometric survey telescope, our result scales to 4.3 mas noise contributed by the atmosphere for 100-second exposures. This is considerably less than previously expected. Thus the limit to ground-based wide field astrometric observations as set by the atmosphere has not yet been reached for long integration times ( $\geq 100$ seconds) because of the relatively large base-level noise of $\approx 0.015$ pixels, which is on the order of 6 to 15 mas, depending on the sampling.

I would like to thank Kitt Peak and Cerro Tololo Observatories for granting observing time. I am grateful to M.I.Zacharias for assistance in observing and reduction of the CCD frames. 


\section{REFERENCES}

Han, I. 1989, AJ 97, 607

Han,I., Gatewood,G.D., 1995, PASP 107, 399

Johnston, K.J., Russell, J.L., de Vegt, C., Zacharias, N., Hindsley, R., Hughes, J., Jauncey, D.L., Reynolds, J.E., Nicholson, G. and Ma, C. 1991, in Reference Systems, Proceedings of IAU Colloq. 127, ed. J.A.Hughes, C.A.Smith and G.H.Kaplan, Washington D.C., US Naval Observatory, p. 123

Kleine, T. 1983, dissertation Univ.of Hamburg

Lindegren, L. 1980, A\&A 89, 41

Monet, D.G. and Monet, A.K.B. 1992, Bull. AAS 24, 1238

Monet, D.G. private communication

Stone,R.C., Monet,D.G., Monet,A.K.B., Walker,R.L., Ables,H.D., Bird,A.R. and Harris,F.H. 1996, AJ 111, 1721

Winter, L. 1996, dissertation Univ. of Hamburg, in preparation

Zacharias,N., de Vegt,C., Winter,L. and Johnston,K. 1995, AJ 110, 3093

Zacharias,N. and Rafferty,T. 1995, Bull.AAS 27, 1302

Zacharias,N. 1997, in preparation for AJ 
Fig. 1.- Precision in the $\mathrm{y}$-coordinate (along $\alpha$ ) for star image profile fits of a typical CCD frame vs. instrumental magnitude. This example is from a 40 second exposure obtained at the KPNO 0.9-meter telescope in 1.6 " seeing. The scale is 0.01 pixel $=6.8$ mas.

Fig. 2.- Variance of $x, y$ transformation $\left(\sigma^{2}=\sigma_{\text {trans }}^{2} / 2\right)$ in $m a s^{2}$ vs. inverse exposure time in $s^{-1}$ for an example from KPNO observations. The filled circles are for the x-coordinate $(\delta)$ and the open boxes are for the $\mathrm{y}$-coordinate $(\alpha)$. Fit results are shown as dotted, full and dashed lines (y only, $\mathrm{x}$ only, both coordinates).

Fig. 3.- Error contribution by the atmosphere to differential astrometry vs. FWHM of image profiles, scaled to the zenith. Full circles and open boxes show results from the KPNO and CTIO telescopes respectively. Only results of the linear $x, y$ transformation model are shown here. 
TABLE 1

Observations and results

\begin{tabular}{|c|c|c|c|c|c|c|c|c|c|}
\hline tel. & $\begin{array}{l}\text { date } \\
\text { (ymd) }\end{array}$ & $\begin{array}{r}\text { z } \\
\text { (degree) }\end{array}$ & $\begin{array}{l}\text { FWHM } \\
(\operatorname{arcsec})\end{array}$ & nexp & $\begin{array}{r}\sigma_{a} \\
(\mathrm{mas})\end{array}$ & $\begin{array}{l}\text { error } \\
\text { (mas) }\end{array}$ & $\begin{array}{r}\sigma_{b} \\
(\mathrm{mas})\end{array}$ & $\begin{array}{l}\text { error } \\
\text { (mas) }\end{array}$ & $\begin{array}{r}\sigma_{a n} \\
(\mathrm{mas})\end{array}$ \\
\hline \multirow[t]{2}{*}{ K } & 940420 & 6 & 2.00 & 4 & 62 & 9 & 7.0 & 0.5 & 5.9 \\
\hline & & & & & 74 & 6 & 7.5 & 0.3 & 7.1 \\
\hline \multirow[t]{2}{*}{ K } & 940422 & 21 & 2.10 & 4 & 39 & 15 & 10.2 & 1.0 & 3.6 \\
\hline & & & & & 50 & 16 & 10.3 & 1.2 & 4.5 \\
\hline \multirow[t]{2}{*}{ K } & 940423 & 17 & 2.65 & 3 & 68 & 13 & 8.2 & 3.0 & 6.3 \\
\hline & & & & & 60 & 15 & 10.1 & 2.9 & 5.5 \\
\hline \multirow[t]{2}{*}{ K } & 941020 & 44 & 1.70 & 4 & 43 & 12 & 9.1 & 0.9 & 3.1 \\
\hline & & & & & 35 & 20 & 12.0 & 1.2 & 2.5 \\
\hline \multirow[t]{2}{*}{ K } & 950613 & 1 & 1.65 & 4 & 31 & 7 & 8.8 & 0.5 & 3.0 \\
\hline & & & & & 36 & 10 & 9.0 & 0.7 & 3.5 \\
\hline \multirow[t]{2}{*}{ K } & 950615 & 12 & 2.10 & 4 & 72 & 6 & 9.1 & 0.5 & 6.8 \\
\hline & & & & & 80 & 7 & 8.9 & 0.6 & 7.5 \\
\hline \multirow[t]{2}{*}{ K } & 950617 & 15 & 1.30 & 4 & 38 & 6 & 7.9 & 0.5 & 3.6 \\
\hline & & & & & 39 & 7 & 8.0 & 0.6 & 3.7 \\
\hline \multirow[t]{2}{*}{ K } & 960105 & 15 & 1.20 & 5 & 39 & 8 & 8.8 & 0.9 & 3.6 \\
\hline & & & & & 45 & 7 & 9.2 & 0.9 & 4.1 \\
\hline \multirow[t]{2}{*}{ C } & 941215 & 17 & 1.35 & 5 & 54 & 7 & 5.8 & 0.7 & 6.0 \\
\hline & & & & & 65 & 10 & 7.4 & 1.1 & 7.3 \\
\hline \multirow[t]{2}{*}{ C } & 950213 & 1 & 1.30 & 4 & 39 & 6 & 6.5 & 0.6 & 4.4 \\
\hline & & & & & 75 & 4 & 9.2 & 0.5 & 8.5 \\
\hline \multirow[t]{2}{*}{$\mathrm{C}$} & 950917 & 1 & 1.60 & 4 & 67 & 6 & 5.9 & 0.8 & 7.6 \\
\hline & & & & & 72 & 15 & 7.8 & 1.6 & 8.2 \\
\hline
\end{tabular}

